\title{
RELATIVISTIC VECTOR BOSONS AND PT-SYMMETRY
}

\author{
J. Smejkal ${ }^{1}$, V. Jakubský ${ }^{2}$, M. Znojil ${ }^{2}$ \\ ${ }^{1}$ Ústav technické a experimentalni fyziky $\check{C} V U T$, \\ Horská, 3a/22, 128 000, Praha 2-Nové Město, Czech Republic

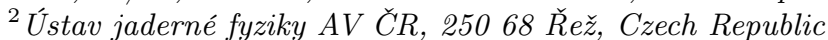

(Received November 27, 2006; received in final form March 1, 2007)

\begin{abstract}
Relativistic massive bosons with spin one are considered in several quantization schemes. In all of them the system is shown to be described by a non-Hermitian Hamiltonian $H \neq H^{\dagger}$ and the helicity operator $\Lambda$. Constructively, we show that in all of the contemplated schemes both these operators $H$ and $\Lambda$ prove simultaneously $\mathcal{P} \mathcal{T}$-symmetric, i. e., pseudo-Hermitian with respect to a certain not too complicated indefinite pseudo-metric operator $\mathcal{P}=\mathcal{P}^{\dagger}$
\end{abstract}

Key words: relativistic vector bosons, quantization, Dirac theory.

PACS number(s): 03.50.Kk, 03.65.Ca, 03.65.Pm

\section{INTRODUCTION}

The majority of textbooks on Quantum Mechanics illustrates the performance of the formalism via a nonrelativistic point particle of mass $m$ which moves in a one-dimensional potential $V(x)$. On the basis of the principle of correspondence the time evolution of such a system is quite often easily described as generated by the ordinary differential self-adjoint Hamiltonian operator $H=\hat{p}^{2}+V(x)$ where $\hat{p}^{2}=-d^{2} / d x^{2}$ in units $\hbar=2 m=1$. Much less attention is usually paid to the possibilities of the transition to relativistic kinematics [1], i.e., to the Klein-Gordon equation for the spinless bosons, the Dirac equation for the simplest fermions, the Maxwell equations for the massless photons as well as the Proca equation for the massive bosons with spin one [2] etc.

It is well known that the correct description of relativistic systems requires the use of full-fledged formalism of relativistic Quantum Field Theory. Another "hidden" reason of the overall reluctance of working with relativistic kinematics even in an approximative regime of Quantum Mechanics is that it often requires a transition to non-self-adjoint generators of the time evolution, i. e., to the Feshbach-Villars Hamiltonian operator $H^{(\mathrm{FV})} \neq\left(H^{(\mathrm{FV})}\right)^{\dagger}$ in the spinless case [3]. Apparently, the naive use of similar Hamiltonians may lead to inconsistencies. For example, negative probabilities of the localization are traditionally mentioned as occurring for the relativistic spinless boson at the point $x$ [4].

The formalism based on the FV-like Hamiltonians $H^{(\mathrm{FV})}$ need not necessarily be mathematically inconsistent $[5,6]$. In fact, the use of $H=H^{(\mathrm{FV})}$ may be reinterpreted as one of the most characteristic applications of the so called PT-symmetric version of Quantum Mechanics as originally proposed by Bender and Boettcher [7]. Thanks to a concentrated effort and subsequent debate (cf. its sample in [8]-[14]) it became clear that even the manifestly non-Hermitian $P T$-symmetric operators of observables $\mathcal{O}$ (characterized, for our present purposes, by their property

$$
\mathcal{O}^{\dagger}=\mathcal{P} \mathcal{O} \mathcal{P}^{-1} \neq \mathcal{O}
$$

with a suitable "indefinite metric" operator $\mathcal{P}$ or with some of its non-metric alternatives [15]) may fit in the overall scheme of Quantum Mechanics.

On this formal background and in the language set already by Scholtz et al in the early nineties [16] it has been recognized that in the relativistic spinless case Hamiltonian $H^{(\mathrm{FV})}$ may be treated as self-adjoint with respect to some less standard inner product in Hilbert space. In other words, the use of the non-Hermitian observables may often be made fully acceptable via the introduction of a certain nontrivial positive definite "physical" metric operator $\Theta \neq I[12]-[16]$.

All the "physical" choices of $\Theta$ must make all the underlying observables (say, $\mathcal{O}=H^{(\mathrm{FV})}$, etc.) self-adjoint in some new $\Theta$-dependent Hilbert space,

$$
\mathcal{O}^{\dagger}=\Theta \mathcal{O} \Theta^{-1} \neq \mathcal{O}, \quad \Theta=\Theta^{\dagger}>0 .
$$

In this sense no modification of the postulates of Quantum Mechanics is needed. Nevertheless in order to avoid confusion the authors of the review [16] recommended that one should speak about quasi-Hermiticity of $\mathcal{O}$ whenever $\Theta \neq I$ in $(2)$.

For the relativistic spinless system the "weakened" Hermiticity property (2) offered a natural explanation of the negative-probability Klein-Gordon puzzle [6]. In what follows we intend to discuss some aspects of the transition to the "next" case of the massive field with the spin one. The main emphasis of our paper will be put on the existence of the $\mathcal{P} \mathcal{T}$-symmetry features of this system and on the emergence of their specific ambiguities which arise in connection with the presence of a nontrivial spin $s>0$ which mediates a coupling of channels [13].

Our present results will be separated into their classical part (section II), quantization part (section III) and $\mathcal{P} \mathcal{T}$-symmetry-related part (section IV). Our observations starting from the classical case may be perceived as separating the Lagrangian and Hamiltonian formulations, with the core of our attention concentrated upon the latter language. Under both the classical and relativistic kinematics our study will be sub-separated 
into the economical "non-Dirac approach" without any constraints (cf. subsections II C and III A or IV A, respectively) and the more common "Dirac approach" using constraints (cf. the respective subsections IID and III B-D or IV B).

In the latter, Dirac-theory context there still exists a certain freedom in the choice of the method of the quantization. Three possibilities, viz., "approach A" (cf. II D 1 or III B), "approach B" (cf. II D 2 or III C) and "approach C" (cf. IID 3 or IIID) are studied in more detail in what follows.

As long as the key purpose of our study lies in an extension of the concept of $\mathcal{P} \mathcal{T}$-symmetry (1) to the equations controlling the motion of the relativistic vector bosons, section IV represents in fact a climax of our considerations. A number of explicit formulae is derived and displayed there including the matrix forms of the Hamiltonian as well as of the related pseudo-metric operator $\mathcal{P}$.

Our last section $\mathrm{V}$ brings a brief summary of our results while Appendix A adds a brief discussion of a few parallels between our present (massive) model and its more common massless (i. e., electromagnetic) counterpart.

\section{BOSONS WITH SPIN ONE}

Our project has been inspired by the description of the massive vector boson system by Taketani, Sakata and Tamm [17] as summarized by Nikitin et al [18]. Our sample of relevant references should not omit Kemmer [19] (in the context of the so-called Kemmer-Duffin-Petiau equation) or Shay and Good and Weinberg [20] (in a slightly different context) and many others, with a nice summary of the field offered by Labenté [21].

\section{A. Lagrangians}

Let us start now our present discussion from the classical case and assume that the dynamics of the classical complex vector field $A$ of mass $m$ is given by its Lagrangian density

$$
\mathcal{L}=m^{2} \bar{A}_{\mu} A^{\mu}-\frac{1}{2}\left(\partial_{\mu} \bar{A}_{\nu}-\partial_{\nu} \bar{A}_{\mu}\right)\left(\partial^{\mu} A^{\nu}-\partial^{\nu} A^{\mu}\right) .
$$

The related action $\mathcal{S}=\int \mathcal{L} d^{4} x$ has to be stationary, $\delta \mathcal{S}=0$. With the current abbreviation $\square=-g^{\mu \nu} \partial_{\mu} \partial_{\nu}$ where $g^{\mu \nu}=\operatorname{diag}(1,-1,-1,-1)$ (and in units such that $c=1$ and $x_{0}=t$ ) this gives the usual Euler-Lagrange equation

$$
\partial_{\mu} F^{\mu \nu}+m^{2} A^{\nu}=0, \quad F^{\mu \nu}=\partial^{\mu} A^{\nu}-\partial^{\nu} A^{\mu}
$$

plus its complex conjugate. Its differentiation and summation reveals that the individual components of the field have to fulfill Klein-Gordon equation subject to the free Klein-Gordon-type constraint,

$$
\left(m^{2}-\square\right) A_{\mu}=0, \quad \partial_{\nu} A^{\nu}=0 .
$$

Consequently, only three of the four components of the field are linearly independent. The equation itself is obviously satisfied by the wave solutions distinguished by the sign $\alpha= \pm$ of the energy,

$$
\begin{aligned}
& A_{\alpha}^{\mu}(x)=N_{\alpha}(p) u_{\alpha}^{\mu}(p) \mathrm{e}^{-i p x}, \quad p_{0}=\alpha \omega, \\
& \omega=\left|\sqrt{\mathbf{p}^{2}+m^{2}}\right| .
\end{aligned}
$$

An additional constraint acquires the form $p u_{\alpha}(p)=0$. For the sake of definiteness the three linearly independent four-vectors $u_{\alpha}(p)$ may be chosen in the following form

$$
\begin{aligned}
& u_{\alpha, 1}=\left(0, \mathbf{u}_{\alpha, 1}\right)^{T}, \quad u_{\alpha, 2}=\left(0, \mathbf{u}_{\alpha, 2}\right)^{T}, \\
& u_{\alpha, 3}=\left(\alpha \frac{|\mathbf{p}|}{\omega}, \frac{\mathbf{p}}{|\mathbf{p}|}\right)^{T} .
\end{aligned}
$$

The space-like components (i. e., polarization vectors) in the first two items are perpendicular to $\mathbf{p}$ while the third one is chosen parallel to $\mathbf{p}$. We may fix

$$
\begin{aligned}
& \mathbf{u}_{\alpha, 1}(\mathbf{p})=\frac{\left(0,-p_{3}, p_{2}\right)^{T}}{\sqrt{p_{3}^{2}+p_{2}^{2}}} \\
& \mathbf{u}_{\alpha, 2}(\mathbf{p})=\frac{\left(p_{2}^{2}+p_{3}^{2},-p_{1} p_{2},-p_{1} p_{3}\right)^{T}}{\sqrt{\left(p_{3}^{2}+p_{2}^{2}\right)^{2}+p_{1}^{2} p_{2}^{2}+p_{1}^{2} p_{3}^{2}}} .
\end{aligned}
$$

Alternatively, the operator of helicity

$$
\hat{h}(\mathbf{p})=\frac{\mathbf{p S}}{|\mathbf{p}|}=\mathbf{n S}=i\left(\begin{array}{ccc}
0 & -n_{3} & n_{2} \\
n_{3} & 0 & -n_{1} \\
-n_{2} & n_{1} & 0
\end{array}\right)
$$

becomes defined in terms of the three generators of the three-dimensional representation of the rotation group,

$$
\begin{aligned}
S_{1} & =\left(\begin{array}{ccc}
0 & 0 & 0 \\
0 & 0 & -i \\
0 & i & 0
\end{array}\right), S_{2}=\left(\begin{array}{ccc}
0 & 0 & i \\
0 & 0 & 0 \\
-i & 0 & 0
\end{array}\right), \\
S_{3} & =\left(\begin{array}{ccc}
0 & -i & 0 \\
i & 0 & 0 \\
0 & 0 & 0
\end{array}\right) .
\end{aligned}
$$

Its action on the vectors $(7),\left[\hat{h} \mathbf{u}_{\alpha, 1}=i \mathbf{u}_{\alpha, 2}, \hat{h} \mathbf{u}_{\alpha, 2}=\right.$ $-i \mathbf{u}_{\alpha, 1}$ and $\hat{h} \mathbf{u}_{\alpha, 3}=0$ (with subscript $\alpha= \pm$ )] prompts an introduction of the helicity-numbered eigenvectors $\mathbf{u}_{\alpha}(p, \pm 1)$

$$
\hat{h} \mathbf{u}_{\alpha}(p, \pm 1)= \pm \mathbf{u}_{\alpha}(p, \pm 1), \quad \hat{h} \mathbf{u}_{\alpha}(p, 0)=0
$$

with an explicit representation $u_{ \pm}(p,+1)=\left(u_{ \pm, 1}+\right.$ $\left.i u_{ \pm, 2}\right) / \sqrt{2}, \quad u_{ \pm}(p,-1)=\left(u_{ \pm, 1}-i u_{ \pm, 2}\right) / \sqrt{2}$ and $u_{ \pm}(p, 0)=u_{ \pm, 3}(p)$. Combining $(5),(7)$ and $(8)$ we get

$$
A_{ \pm, h}^{\mu}=N_{ \pm, h} u_{ \pm}^{\mu}(p, h) \mathrm{e}^{i \mathbf{p x} \mp i \omega t}
$$

i. e., waves traveling at a fixed energy and helicity. 


\section{B. Hamiltonians}

There exist, basically, two ways of the derivation of the description of the time evolution of the vector boson fields from the first principles. In one of them one eliminates all the (redundant) degrees of freedom from the very beginning. We shall call this approach "economical" because the number of its field "parameters" is, in some sense, minimal and equal to the number of the degrees of freedom of the field (cf. also subsection II C for more details).

An alternative recipe (cf. the subsequent subsection IID) carries the name of Dirac [22]. It employs the language of constraints and its key merit may be seen in its more formal, "algorithmic" character. It offers a virtually unambiguous recipe for a systematic treatment of the field in question. Both its physical and non-physical components are treated "on an equal footing".

The key purpose of our present paper lies in the discussion of the latter two approaches. We intend to illustrate (by construction!) that both of them remain equivalent on the classical level while a number of open questions can arise during their quantization.

Before we fully concentrate on the questions of specific differences between quantization recipes for vector bosons (in which the classical fields should be replaced by operators) we intend to emphasize that several important technicalities must already be re-analyzed in a preparatory step, on the classical level. On this level the first relevant aspect of the problem may be determined immediately as lying in the existence of the "economical" possibility which looks nonstandard and which seems to be in an apparent conflict with the more modern formulations of the problem.

In the modern (usually called Dirac's) theory the quantization is treated using the BRST and BatalinVilkovisky methods [23]. For a more detailed elucidation of the topic the reader might consult related lit- erature where the quantization of the relativistic (free) particles is reminiscent of Feynman's fifth time formalism and where the theory involves the quantization of the reparametrization-invariant models on the world line, etc.

The latter approach leads to the Klein-Gordon equation emerging as a constraint (analogous to the Gauss law in QED) and it opens new perspectives, i. a., in supersymmetry. At the same time it does not represent the only possibility and it may often be complemented by the less subtle approach where the Klein-Gordon equation results from the "economical" process where one starts from the elimination of all the redundant degrees of freedom. Thus, once we succeed in a minimization of the number of the fields we are not forced to impose any auxiliary constraints. We shall add more details in paragraph II C.

One could stress that the minimization strategy circumvents (or at least clarifies) certain shortcomings of the use of Dirac's eight-dimensional Hamiltonians by their replacement by compact, six-dimensional "economical" alternatives. Conversely, specific merits of the eightdimensional formulae will be recollected in paragraph IID. We shall clarify there the distinction between several alternative ways of a detailed application of Dirac's recipe. We shall distinguish between a certain "approach A" (cf. paragraph IID 1 for more details), "approach B" (cf. paragraph II D 2) and "approach C" (cf. paragraph IID 3). We believe that in this way an enhanced transparency will be assigned not only to the essence of the (second-class) constraints but also to the subsequent questions of the quantization.

\section{C. "Economical" formulation}

Using the standard recipe and taking $A_{\mu}$ and $\bar{A}_{\mu}$ as generalized coordinates we get the generalized momenta,

$$
\begin{array}{ll}
\Pi_{m}=\frac{\partial \mathcal{L}}{\partial\left(\partial_{0} A_{m}\right)}=\partial_{0} \bar{A}_{m}-\partial_{m} \bar{A}_{0}, & \Pi_{0}=\frac{\partial \mathcal{L}}{\partial\left(\partial_{0} A_{0}\right)}=0, \\
\bar{\Pi}_{m}=\frac{\partial \mathcal{L}}{\partial\left(\partial_{0} \bar{A}_{m}\right)}=\partial_{0} A_{m}-\partial_{m} A_{0}, & \bar{\Pi}_{0}=\frac{\partial \mathcal{L}}{\partial\left(\partial_{0} \bar{A}_{0}\right)}=0 .
\end{array}
$$

We see that the time-coordinate momenta $\Pi_{0}$ and $\bar{\Pi}_{0}$ can be omitted whereas their respective field conjugates can be eliminated using the equations of motion (3),

$$
A_{0}=\frac{1}{m^{2}}\left(\partial_{m} \bar{\Pi}_{m}\right), \quad \bar{A}_{0}=\frac{1}{m^{2}}\left(\partial_{m} \Pi_{m}\right) .
$$

After a straightforward calculation the density of the Hamiltonian of our classical vector field can be found,

$$
\begin{aligned}
& \mathcal{H}=\Pi_{m}\left(\partial_{0} A_{m}\right)+\bar{\Pi}_{m}\left(\partial_{0} \bar{A}_{m}\right)-\mathcal{L} \\
& =\bar{\Pi}_{m} \Pi_{m}+\frac{1}{m^{2}}\left(\partial_{m} \bar{\Pi}_{m}\right)\left(\partial_{n} \Pi_{n}\right)+m^{2} \bar{A}_{m} A_{m}
\end{aligned}
$$

$$
+\left(\partial_{m} \bar{A}_{n}\right)\left(\partial_{m} A_{n}\right)-\left(\partial_{m} \bar{A}_{m}\right)\left(\partial_{n} A_{n}\right)
$$

Classically, Euler-Lagrange equations (4) correspond to the dynamical equations

$$
\dot{A}_{m}=\left\{H, A_{m}\right\}_{\mathrm{P}}, \quad \dot{\Pi}_{m}=\left\{H, \Pi_{m}\right\}_{\mathrm{P}}
$$

where $\{., .\}_{\mathrm{P}}$ denotes the Poisson bracket and where $H=\int \mathcal{H} d \mathbf{x}$. 


\section{Dirac theory using constraints}

We already mentioned that there are several possibilities of the strictly formal transition from Lagrangians to Hamiltonians. Sometimes, one has to interpret the impossibility of the elimination of generalized velocities as an emergence of certain phase-space constraints (to be denoted as $\theta$ ). They are called "primary constraints" and will be marked by the superscript ${ }^{(I)}$. We shall have to deal with a pair of them in our vector-boson model.

In addition, the natural requirement of the timeindependence of the constraints $\theta^{(\mathrm{I})}$ leads, in principle, to the emergence of further series of constraints denoted as $\theta^{(\mathrm{II})}$. They are called "secondary" and we will have a pair of them in what follows.

In approach "A" of the formalism (cf. paragraph II D 1 below), the Hamiltonians will be constructed, using the technique of the Lagrange multipliers, in a way which employs the primary constraints $\theta^{(\mathrm{I})}$ only. In approach "B" of paragraph IID 2, the knowledge of all the constraints $\theta^{(\mathrm{I})}$ and $\theta^{(\mathrm{II})}$ will be employed giving an alternative family of the Hamiltonian functions. Finally, approach "C" of paragraph II D 3 will re-define the brackets and enable us to prepare and perform the quantization in an internally most consistent and compact manner.

\section{Approach $A$}

In approach "A" one has to apply the Lagrange multipliers in order to respect the existence of the two primary constraints. These constraints read

$$
\theta^{(\mathrm{I})}=\Pi_{0}=0, \quad \bar{\theta}^{(\mathrm{I})}=\bar{\Pi}_{0}=0
$$

and emerge in the process of the transformation of the Lagrangian into the Hamiltonian (cf. also the formulae of eq. (11)). An "extended" Hamiltonian $\mathcal{H}^{(\mathrm{A})}$ will be then sought as containing a pair of the Lagrange multipliers $\lambda^{(\mathrm{I})}$ and $\bar{\lambda}^{(\mathrm{I})}$,

$$
\mathcal{H}^{(\mathrm{A})}=\mathcal{H}^{0}+\lambda^{(\mathrm{I})} \theta^{(\mathrm{I})}+\bar{\lambda}^{(\mathrm{I})} \bar{\theta}^{(\mathrm{I})} .
$$

The symbol $\mathcal{H}^{0}$ stands for the standard, non-extended Hamiltonian function,

$$
\begin{aligned}
\mathcal{H}^{0} & =\Pi_{m}\left(\partial_{0} A_{m}\right)+\bar{\Pi}_{m}\left(\partial_{0} \bar{A}_{m}\right)-\mathcal{L}= \\
& =\bar{\Pi}_{m} \Pi_{m}+m^{2} \bar{A}_{m} A_{m}-m^{2} \bar{A}_{0} A_{0}+\left(\partial_{m} \bar{A}_{n}\right)\left(\partial_{m} A_{n}\right) \\
& -\left(\partial_{m} \bar{A}_{m}\right)\left(\partial_{n} A_{n}\right)+\Pi_{m}\left(\partial_{m} A_{0}\right)+\bar{\Pi}_{m}\left(\partial_{m} \bar{A}_{0}\right) .
\end{aligned}
$$

In the next step one demands the time independence of the primary constraints,

$$
\left\{H^{(\mathrm{A})}, \theta^{(\mathrm{I})}\right\}_{\mathrm{P}}=0, \quad\left\{H^{(\mathrm{A})}, \bar{\theta}^{(\mathrm{I})}\right\}_{\mathrm{P}}=0,
$$

where

$$
H^{(\mathrm{A})}=\int d \vec{x} \mathcal{H}^{(\mathrm{A})} .
$$

In our present model such a requirement gives rise to a mere doublet of secondary constraints,

$$
\begin{aligned}
& \theta^{(\mathrm{II})}=-m^{2} \bar{A}_{0}-\left(\partial_{m} \Pi_{m}\right)=0, \\
& \bar{\theta}^{(\mathrm{II})}=-m^{2} A_{0}-\left(\partial_{m} \bar{\Pi}_{m}\right)=0 .
\end{aligned}
$$

In the subsequent step we have to demand time independence of the secondary constraints, too. In a way paralleling eq. (17) we obtain the Lagrange multipliers $\lambda^{(\mathrm{I})}$ and $\bar{\lambda}^{(\mathrm{I})}$ as a solution. In this way we arrive at the final Hamiltonian

$$
\mathcal{H}^{(\mathrm{A})}=\mathcal{H}^{0}+\Pi_{0}\left(\partial_{m} A_{m}\right)+\bar{\Pi}_{0}\left(\partial_{m} \bar{A}_{m}\right) .
$$

The process of construction guarantees that all the equations of motion respect all the primary and secondary constraints.

\section{Approach B}

In the previous approach $\mathrm{A}$, the secondary constraints were clearly discriminated in comparison with the primary ones. Fortunately, in a way outlined, e.g., in the popular textbook [24] we can also introduce all the constraints in the Hamiltonian simultaneously, regardless of their origin.

First steps of such a slightly more sophisticated approach to the construction of Hamiltonians are exactly the same as in approach A. The differences appear after one obtains all the secondary constraints. Having collected all the constraints $\theta^{(\mathrm{I})}$ and $\theta^{(\mathrm{II})}$ one decides to employ them all in the recipe using Lagrange multipliers. This means that our new postulated Hamiltonian $\mathcal{H}^{(\mathrm{B})}$ will read

$$
\begin{aligned}
\mathcal{H}^{(\mathrm{B})} & =\mathcal{H}^{0}+\lambda^{(\mathrm{I})} \theta^{(\mathrm{I})}+\bar{\lambda}^{(\mathrm{I})} \bar{\theta}^{(\mathrm{I})} \\
& +\lambda^{(\mathrm{II})} \theta^{(\mathrm{II})}+\bar{\lambda}^{(\mathrm{II})} \bar{\theta}^{(\mathrm{II})}
\end{aligned}
$$

where the symbols $\lambda^{(\mathrm{II})}$ and $\bar{\lambda}^{(\mathrm{II})}$ denote two new Lagrange multipliers attached to the secondary constraints. All the values of the lambda-multipliers emerging in eq. (21) may be again extracted from the requirement of the time independence,

$$
\begin{aligned}
& \left\{H^{(\mathrm{B})}, \theta\right\}_{\mathrm{P}}=0, \quad \theta \in\left\{\theta^{(\mathrm{I})}, \bar{\theta}^{(\mathrm{I})}, \theta^{(\mathrm{II})}, \bar{\theta}^{(\mathrm{II})}\right\}, \\
& H^{(\mathrm{B})}=\int d \vec{x} \mathcal{H}^{(\mathrm{B})} .
\end{aligned}
$$

The resulting Hamiltonian

$$
\begin{aligned}
& \mathcal{H}^{(\mathrm{B})}=\mathcal{H}^{0}+\Pi_{0}\left(\partial_{m} A_{m}\right)+\bar{\Pi}_{0}\left(\partial_{m} \bar{A}_{m}\right) \\
& +\frac{2}{m^{2}}\left[m^{2} \bar{A}_{0}+\left(\partial_{m} \Pi_{m}\right)\right]\left[m^{2} A_{0}+\left(\partial_{m} \bar{\Pi}_{m}\right)\right]
\end{aligned}
$$

will prescribe the motion equivalent to the one obtained within approach $\mathrm{A}$, on the classical level at least. 


\section{Approach $C$}

A key shortcoming of both the above approaches A and $\mathrm{B}$ (which lead to the same equations of motion as obtained in the "economical" setting) is that they prove less convenient for quantization purposes. Apparently, this would make Dirac's theory manifestly disqualified in comparison with the economical approach. Fortunately, it is possible (as well as not too difficult) to re-adapt the Dirac procedure for quantization purposes.

In the first step we calculate the Poisson brackets for all the constraints and define a certain auxiliary matrix $\mathcal{M}$,

$$
\begin{aligned}
& (\mathcal{M}(\vec{x}, \vec{y}))_{J, K}=\left\{\theta^{(J)}(\vec{x}), \theta^{(K)}(\vec{y})\right\}_{\mathrm{P}}, \\
& J, K=1,2,3,4
\end{aligned}
$$

where we renumbered

$$
\begin{array}{ll}
\theta^{(1)}=\theta^{(\mathrm{I})}, & \theta^{(2)}=\theta^{(\mathrm{II})}, \\
\theta^{(3)}=\bar{\theta}^{(\mathrm{I})}, & \theta^{(4)}=\bar{\theta}^{(\mathrm{II})} .
\end{array}
$$

We find that

$$
\mathcal{M}_{J K}(\vec{x}, \vec{y})=\mathbf{M}_{J K} \delta^{(3)}(\vec{x}-\vec{y})
$$

with the boldface symbol $\mathbf{M}$ denoting an antidiagonal matrix,

$$
\mathbf{M}=\left(\begin{array}{cccc}
0 & 0 & 0 & m^{2} \\
0 & 0 & -m^{2} & 0 \\
0 & m^{2} & 0 & 0 \\
-m^{2} & 0 & 0 & 0
\end{array}\right), \quad \mathbf{M}^{-1}=-\frac{1}{m^{4}} \mathbf{M}
$$

As long as matrix $\mathbf{M}$ is non-singular and invertible we can easily insert

$$
\mathcal{M}_{J K}^{-1}(\vec{x}, \vec{y})=\mathbf{M}_{J K}^{-1} \delta^{(3)}(\vec{x}-\vec{y})
$$

in an explicit definition of the Lagrange multipliers of approach B and obtain

$$
\lambda^{(J)}=-\sum_{K=1}^{4}\left\{H^{0}, \theta^{(K)}\right\}_{\mathrm{P}} \mathbf{M}_{K J}^{-1} .
$$

The numbering of $\lambda_{\mathrm{s}}$ is inherited from $\theta_{\mathrm{s}}$ (cf. eq. (25)) and gives the final compact formula for the Hamiltonian,

$$
\begin{aligned}
& \mathcal{H}^{(\mathrm{B})}=\mathcal{H}^{0}-\sum_{J, K=1}^{4}\left\{H^{0}, \theta^{(K)}\right\}_{\mathrm{P}} \mathbf{M}_{K J}^{-1} \theta^{(J)}, \\
& H^{0}=\int d \vec{x} \mathcal{H}^{0} .
\end{aligned}
$$

However, our work is not finished yet since the consistency of the recipe still requires a replacement of the Poisson brackets by the so-called Dirac brackets,

$$
\begin{aligned}
\{X, Y\}_{\mathrm{D}} & =\{X, Y\}_{\mathrm{P}}-\sum_{J, K=1}^{4} \int d \vec{w} d \vec{z}\left\{X, \theta^{(J)}(\vec{w})\right\}_{\mathrm{P}} \\
& \times \mathcal{M}_{J K}^{-1}(\vec{w}, \vec{z})\left\{\theta^{(K)}(\vec{z}), Y\right\}_{\mathrm{P}} .
\end{aligned}
$$

Fortunately, one can show very easily that the equations of motion which result from approach B are practically equivalent to those which can be obtained from eq. (31). Roughly speaking, there emerge differences reducible to a certain combination of terms which would vanish due to the constraints. Ref. [24] may be consulted for details.

The main advantage of the replacement of the Poisson brackets by their Dirac alternative is that the Dirac brackets annihilate all the constraints,

$$
\left\{X, \theta^{(J)}\right\}_{\mathrm{D}}=0 \quad \text { for } J \in\{1,2,3,4\} \text { and } \forall X \text {. }
$$

Such a property will prove extremely useful during quantization since the use of the Dirac brackets (in place of the Poisson ones) enables us to work with the "core" Hamiltonian $\mathcal{H}^{0}$ only.

\section{QUANTIZATION IN HEISENBERG PICTURE}

\section{A. "Economical" formulation}

In the Heisenberg picture the quantization of the system yields a replacement of the generalized coordinates $A_{m}$ and $\Pi_{n}$ and of their complex conjugates by the noncommutative operators which satisfy the canonical equaltime commutation relations,

$$
\left[A_{m}(\mathbf{x}), \Pi_{n}(\mathbf{y})\right]=i \delta_{n m} \delta^{(3)}(\mathbf{x}-\mathbf{y}),
$$

$\left[\bar{A}_{m}(\mathbf{x}), \bar{\Pi}_{n}(\mathbf{y})\right]=i \delta_{n m} \delta^{(3)}(\mathbf{x}-\mathbf{y}), \quad n, m=1,2,3$.

They are- up to the factor $i$-parallel to the Poissonbracket rules.

The time-evolution of the operators is determined by the modified equations (13),

$$
\dot{A}_{k}=-i\left[H, A_{k}\right]=-\bar{\Pi}_{k}+\frac{1}{m^{2}} \partial_{k}\left(\partial_{n} \bar{\Pi}_{n}\right),
$$

$$
\dot{\bar{\Pi}}_{k}=-i\left[H, \bar{\Pi}_{k}\right]=m^{2} A_{k}-\triangle A_{k}+\partial_{k}\left(\partial_{n} A_{n}\right),
$$

$$
\dot{\bar{A}}_{k}=-i\left[H, \bar{A}_{k}\right]=-\Pi_{k}+\frac{1}{m^{2}} \partial_{k}\left(\partial_{n} \Pi_{n}\right),
$$

$$
\dot{\Pi}_{k}=-i\left[H, \Pi_{k}\right]=m^{2} \bar{A}_{k}-\triangle \bar{A}_{k}+\partial_{k}\left(\partial_{n} \bar{A}_{n}\right) .
$$

An arrangement of $A$ and $\bar{\Pi}$ in a single six-component set $\Psi^{T}=(m \mathbf{A}, i \overline{\mathbf{\Pi}})$ is sometimes being used [25]. 


\section{B. Dirac theory - approach A}

In place of the six-dimensional Hamiltonians $H$ pertaining to the preceding approach let us now switch to the so-called Dirac's approach $[22,24]$ where, as we have already outlined, certain more-dimensional Hamiltonians with redundant components are being used.

The form of the commutators should result from their Poisson-bracket predecessors. Nevertheless, we have to keep in mind the existence of the additional, "redundant" degrees of freedom now. Thus, an additional pair of operators with the non-trivial commutators emerges,

$$
\begin{gathered}
{\left[A_{0}(\mathbf{x}), \Pi_{0}(\mathbf{y})\right]=i \delta^{(3)}(\mathbf{x}-\mathbf{y}),} \\
{\left[\bar{A}_{0}(\mathbf{x}), \bar{\Pi}_{0}(\mathbf{y})\right]=i \delta^{(3)}(\mathbf{x}-\mathbf{y}) .}
\end{gathered}
$$

Performing all the calculations we may collect an extended set of equations,

$$
\begin{aligned}
\dot{A}_{k} & =-i\left[H^{(\mathrm{A})}, A_{k}\right]=-\bar{\Pi}_{k}-\left(\partial_{k} A_{0}\right), \\
\dot{\Pi}_{k} & =-i\left[H^{(\mathrm{A})}, \Pi_{k}\right]=m^{2} \bar{A}_{k} \\
& -\triangle \bar{A}_{k}+\partial_{k}\left(\partial_{n} \bar{A}_{n}\right)-\left(\partial_{k} \Pi_{0}\right), \\
\dot{A}_{0} & =-i\left[H^{(\mathrm{A})}, A_{0}\right]=-\left(\partial_{m} A_{m}\right), \\
\dot{\Pi}_{0} & =-i\left[H^{(\mathrm{A})}, \Pi_{0}\right]=-m^{2} \bar{A}_{0}-\left(\partial_{m} \Pi_{m}\right) .
\end{aligned}
$$

For the sake of brevity we do not display the complementary, complex conjugate version of these equations.

\section{Dirac theory - approach B}

The Hamiltonian $H^{(\mathrm{B})}$ enters formulae

$$
\begin{aligned}
\dot{A}_{k} & =-i\left[H^{(\mathrm{B})}, A_{k}\right]=-\bar{\Pi}_{k}+\frac{2}{m^{2}} \partial_{k}\left(\partial_{n} \bar{\Pi}_{n}\right)+\left(\partial_{k} A_{0}\right), \\
\dot{\Pi}_{k} & =-i\left[H^{(\mathrm{B})}, \Pi_{k}\right]=m^{2} \bar{A}_{k} \\
& -\triangle \bar{A}_{k}+\partial_{k}\left(\partial_{n} \bar{A}_{n}\right)-\left(\partial_{k} \Pi_{0}\right) \\
\dot{A}_{0} & =-i\left[H^{(\mathrm{B})}, A_{0}\right]=-\left(\partial_{m} A_{m}\right) \\
\dot{\Pi}_{0} & =-i\left[H^{(\mathrm{B})}, \Pi_{0}\right]=+m^{2} \bar{A}_{0}+\left(\partial_{m} \Pi_{m}\right) .
\end{aligned}
$$

In essence, the practical consequences of the procedure remain virtually the same as before.

\section{Dirac theory - approach C}

A major change comes with the necessity of using the "Dirac-originating" commutators in place of the "Poissonoriginating" ones. This leads to the following two nontrivial rules

$$
\begin{aligned}
& {\left[A_{0}(\mathbf{x}), \Pi_{0}(\mathbf{y})\right]=0} \\
& {\left[A_{0}(\mathbf{x}), \bar{A}_{m}(\mathbf{y})\right]=-i \frac{\partial}{\partial y_{m}} \delta^{(3)}(\mathbf{x}-\mathbf{y})}
\end{aligned}
$$

(cf. relations of eqs. (33) and (35)). In the light of the latter "distortion" of the commutators we are allowed to employ Hamiltonian $H^{0}$ and to arrive at the set of equations

$$
\begin{aligned}
\dot{A}_{k} & =-i\left[H^{(0)}, A_{k}\right]=-\bar{\Pi}_{k}-\frac{1}{m^{2}} \partial_{k}\left(\partial_{n} \bar{\Pi}_{n}\right)-2\left(\partial_{k} A_{0}\right) \\
\dot{\Pi}_{k} & =-i\left[H^{(0)}, \Pi_{k}\right]=m^{2} \bar{A}_{k} \\
& -\triangle \bar{A}_{k}+\partial_{k}\left(\partial_{n} \bar{A}_{n}\right) \\
\dot{A}_{0} & =-i\left[H^{(0)}, A_{0}\right]=-\left(\partial_{m} A_{m}\right) \\
\dot{\Pi}_{0} & =-i\left[H^{(0)}, \Pi_{0}\right]=0
\end{aligned}
$$

We may observe and conclude that all the available recipes are equivalent because they differ only by the terms which are proportional to certain combinations of the constraints.

\section{TIME-EVOLUTION AND $\mathcal{P} \mathcal{T}$-SYMMETRY}

\section{A. Six-dimensional Hamiltonian}

After the "economical" quantization we arrive at the formal Schrödinger equation

$$
i \frac{\partial}{\partial t}|\Psi\rangle=H|\Psi\rangle
$$

which prescribes the time-evolution of the operators in our system. The quantum Hamiltonian $H=H^{(\mathrm{VB})}$ of the vector boson is the matrix

$$
H=\left(\begin{array}{cc}
0 & -m+\frac{\operatorname{grad} d i v}{m} \\
-m+\frac{\nabla^{2}}{m}-\frac{\operatorname{grad} d i v}{m} & 0
\end{array}\right) .
$$

(cf. [25]). In the coordinate representation it has the $6 \times 6$ dimensional and manifestly non-Hermitian partitioned matrix structure,

$$
H \sim\left(\begin{array}{cccccc}
0 & 0 & 0 & \partial_{1}^{2}-m^{2} & \partial_{1} \partial_{2} & \partial_{1} \partial_{3} \\
0 & 0 & 0 & \partial_{2} \partial_{1} & \partial_{2}^{2}-m^{2} & \partial_{2} \partial_{3} \\
0 & 0 & 0 & \partial_{3} \partial_{1} & \partial_{3} \partial_{2} & \partial_{3}^{2}-m^{2} \\
-\partial_{1}^{2}-\omega^{2} & -\partial_{1} \partial_{2} & -\partial_{1} \partial_{3} & 0 & 0 & 0 \\
-\partial_{2} \partial_{1} & -\partial_{2}^{2}-\omega^{2} & -\partial_{2} \partial_{3} & 0 & 0 & 0 \\
-\partial_{3} \partial_{1} & -\partial_{3} \partial_{2} & -\partial_{3}^{2}-\omega^{2} & 0 & 0 & 0
\end{array}\right)
$$


This is a $\mathcal{P}$-pseudo-Hermitian operator,

$$
H^{\dagger}=\mathcal{P} H \mathcal{P}^{-1} \neq H, \quad \mathcal{P}=\left(\begin{array}{cc}
0 & I_{3} \\
I_{3} & 0
\end{array}\right) .
$$

The six independent eigenvectors with eigenvalues $\pm p_{0}$ may be most easily constructed in momentum representation. For this purpose we introduce another observable which commutes with $H$,

$$
\begin{gathered}
\Lambda_{i, j}(\mathbf{p})=\Lambda_{i+3, j+3}(\mathbf{p})=-\frac{1}{2}+\frac{\frac{3}{2} p_{i} p_{j}+i|\mathbf{p}| \varepsilon_{i j k} p_{k}}{\mathbf{p}^{2}} \\
\hat{\Lambda}_{i, j+3}(\mathbf{p})=\hat{\Lambda}_{i+3, j}(\mathbf{p})=0, \quad i, j=1,2,3 .
\end{gathered}
$$

This is a block diagonal operator which distinguishes the spin projection into momentum direction. In terms of physics it corresponds to the helicity of our vector bosons. It is easy to verify that the latter operator is also pseudo-Hermitian with respect to $\mathcal{P}$,

$$
\Lambda^{\dagger}=\mathcal{P} \Lambda \mathcal{P}^{-1}
$$

Denoting $\left| \pm p_{0}, h\right\rangle=\Psi^{( \pm)}(\mathbf{p}, h)|\mathbf{p}\rangle$ we obtain a complete right-action Schrödinger equation for the fixed-helicity states,

$$
\begin{aligned}
& H| \pm \omega, h\rangle= \pm \omega| \pm \omega, h\rangle, \\
& \hat{\Lambda}| \pm \omega, h\rangle=h| \pm \omega, h\rangle, \quad h= \pm 1, \quad 0
\end{aligned}
$$

as well as a $\mathrm{R}$ similar relation for the action of our observables to the left,

$$
\begin{aligned}
& \langle\langle \pm \omega, h| H= \pm \omega\langle\langle \pm \omega, h|, \\
& \langle\langle \pm \omega, h| \hat{\Lambda}=h\langle\langle \pm \omega, h|, \quad h= \pm 1,0 .
\end{aligned}
$$

In a way described more thoroughly in ref. [13] the pseudo-Hermicity of both our observables can immediately be used to express the double-ket-marked eigenvectors of the conjugate-operator pair of $H^{\dagger}$ and $\Lambda^{\dagger}$ in terms of the single-ket-marked eigenvectors of the pair of operators $H$ and $\Lambda$,

$$
| \pm \omega, h\rangle\rangle=\mathcal{P}| \pm \omega, h\rangle \varrho_{p_{0}, h} .
$$

One can normalize the vectors $\left|p_{0}, h\right\rangle$ and $\left.\left|p_{0}^{\prime}, h^{\prime}\right\rangle\right\rangle$ in such a way that they form a biorthonormal set,

$$
\left\langle\left\langle p_{0}, h \mid p_{0}^{\prime}, h^{\prime}\right\rangle=\delta\left(p_{0}-p_{0}^{\prime}\right) \delta_{h h^{\prime}}\right.
$$

Naturally, one could also switch to the language of the unit vectors $\mathbf{e}_{1}, \mathbf{e}_{2}$ which are chosen as mutually perpendicular, $\left(\mathbf{e}_{1}, \overline{\mathbf{e}}_{2}\right)=0$, and perpendicular to the momentum vector $\mathbf{p}$. Then, one can decompose any positiveor negative-energy amplitude into its three separate perpendicular components,

$$
\mathbf{A}^{( \pm)}(\mathbf{p})=a_{1}^{( \pm)}(\mathbf{p}) \mathbf{e}_{1}+a_{2}^{( \pm)}(\mathbf{p}) \mathbf{e}_{2}+a_{3}^{( \pm)}(\mathbf{p}) \frac{\mathbf{p}}{|\mathbf{p}|}
$$

and introduce the three linearly independent auxiliary amplitudes $A_{i}^{( \pm)}(p)$ as perpendicular to the momentum four-vector,

$$
\begin{aligned}
& A_{1}^{( \pm)}(\mathbf{p})=\left(0, \mathbf{e}_{1}\right), \quad A_{2}^{( \pm)}(\mathbf{p})=\left(0, \mathbf{e}_{2}\right), \\
& A_{3}^{( \pm)}=\left(\frac{|\mathbf{p}|}{p_{0}}, \frac{\mathbf{p}}{|\mathbf{p}|}\right) .
\end{aligned}
$$

This enables us to abbreviate

$$
\begin{gathered}
A^{( \pm)}(p, 1)=\frac{A_{1}^{( \pm)}(p)+i A_{2}^{( \pm)}(p)}{\sqrt{2}}, \\
A^{( \pm)}(p, 2)=\frac{A_{1}^{( \pm)}(p)-i A_{2}^{( \pm)}(p)}{\sqrt{2}}, \\
A^{( \pm)}(p, 3)=A_{3}^{( \pm)}(p)
\end{gathered}
$$

and

$$
\Psi_{k}^{( \pm)}(p, j)=\left\{\begin{array}{ll}
m A_{k}^{( \pm)}(p, j), & k=1,2,3 \\
\mp \omega A_{k}^{( \pm)}(p, j)+p_{k} A_{0}^{( \pm)}(p, j), & k=4,5,6
\end{array}, \quad j=1,2,3\right.
$$

In this way the definition of the eigenvectors is related again to the "more traditional" amplitudes of the field in momentum representation.

\section{B. Alternative eight-dimensional Hamiltonians}

In the Dirac case we can write Hamiltonians in the partitioned matrix form, too. All such matrices will be eightdimensional.

In approach $\mathrm{A}$, the matrix form of the Hamiltonian is compatible with the related equations of motion (36),

$$
H^{(\mathrm{A})}=\left(\begin{array}{cccc}
0 & -m & -\operatorname{grad} & 0 \\
-m+\frac{\nabla^{2}}{m}-\frac{\operatorname{grad} \operatorname{div}}{m} & 0 & 0 & \operatorname{grad} \\
-\operatorname{div} & 0 & 0 & 0 \\
0 & \operatorname{div} & m & 0
\end{array}\right) .
$$


It is amusing to notice that approach B will give the matrix Hamiltonian in a slightly different form,

$$
H^{(\mathrm{B})}=\left(\begin{array}{cccc}
0 & -m+\frac{2 \text { grad div }}{m} & \operatorname{grad} & 0 \\
-m+\frac{\nabla^{2}}{m}-\frac{\text { grad div }}{m} & 0 & 0 & \operatorname{grad} \\
-\operatorname{div} & 0 & 0 & 0 \\
0 & -\operatorname{div} & -m & 0
\end{array}\right)
$$

(cf. equations of motion (37)). Still another version of the Hamiltonian will emerge from approach C,

$$
H^{0}=\left(\begin{array}{cccc}
0 & -m-\frac{\text { grad div }}{m} & -2 \operatorname{grad} & 0 \\
-m+\frac{\nabla^{2}}{m}-\frac{\operatorname{grad} d i v}{m} & 0 & 0 & 0 \\
-\operatorname{div} & 0 & 0 & 0 \\
0 & 0 & 0 & 0
\end{array}\right)
$$

(cf. equations of motion (39)). All the latter matrices are non-Hermitian. However, they are also $\mathcal{P} \mathcal{T}$-symmetric in the way which employs the same, constant and partitioned, matrix operator

$$
\mathcal{P}=\left(\begin{array}{cccc}
0 & I_{3} & 0 & 0 \\
I_{3} & 0 & 0 & 0 \\
0 & 0 & 0 & -1 \\
0 & 0 & -1 & 0
\end{array}\right)
$$

in all the three contexts. It is worth emphasizing that the use of the Hamiltonian pertaining to the Dirac approach C seems definitely preferable from the point of view of quantization.

\section{SUMMARY}

Once we assume that the pair of a quantum Hamiltonian $H$ and some auxiliary "observable" $\Lambda$ are independent and diagonalizable non-Hermitian operators with discrete spectra, we may study this pair as a special mathematical realization of a $\mathcal{P} \mathcal{T}$-symmetric quantum model [13]. In our present paper we paid particular attention to the related necessity of the explicit knowledge of a sufficiently simple auxiliary generalized parity operator $\mathcal{P}$. In this sense we have shown here that the classical Proca's field offers a new and feasible example of a $\mathcal{P} \mathcal{T}$ symmetric system, this time with an immediate physical vector-boson interpretation.

\section{A. Proca system with pseudo-Hermitian observables}

Let us summarize our preceding text as a constructive presentation of the triplet of operators $H, \Lambda$ and $\mathcal{P}$ on a Hilbert space $\mathcal{V}$ which fulfills the relations

$$
H^{\dagger}=\mathcal{P} H \mathcal{P}^{-1}, \quad \Lambda^{\dagger}=\mathcal{P} \Lambda \mathcal{P}^{-1}
$$

where $\mathcal{P}$ is an extremely simple though nontrivial and positively indefinite Hermitian automorphism on $\mathcal{V}$.

In the related literature, the $\mathcal{P} \mathcal{T}$-symmetric operators $H$ and $\Lambda$ are also often called pseudo-Hermitian or
$\mathcal{P}$-pseudo-Hermitian. One of the most important consequences of their $\mathcal{P} \mathcal{T}$-symmetry is known to lie in the facilitated possibility of the construction of the physical or "representation" Hilbert space and a metric using the factorization ansatz $\Theta=\mathcal{C P}$ where $\mathcal{C}$ may be called a "charge" [12].

Let us repeat that having assumed the simultaneous diagonalizability and a non-degeneracy of the (real or complex) spectra of both $H$ and $\Lambda$ we can, at least in principle, find their mutually bi-orthogonal left and right eigenvectors,

$$
\begin{gathered}
H|n, h\rangle=E_{n}|n, h\rangle, \quad\left\langle\langle m, h| H=\left\langle\langle m, h| E_{m},\right.\right. \\
\Lambda|n, h\rangle=h|n, h\rangle, \quad\langle\langle m, h| \Lambda=\langle\langle m, h| h,
\end{gathered}
$$

as well as their formal spectral representation,

$$
\begin{aligned}
H & =\sum_{n}|n, h\rangle \frac{E_{n}}{\langle\langle n, h \mid n, h\rangle}\langle\langle n, h|, \\
\Lambda & =\sum_{h}|n, h\rangle \frac{h}{\langle\langle n, h \mid n, h\rangle}\langle\langle n, h| .
\end{aligned}
$$

Due to (49), the left and right eigenvectors are mutually related by $\mathcal{P}$

$$
\left.\mathcal{P}|n, h\rangle \sim \rho_{n, h}|n, h\rangle\right\rangle,
$$

where the "quasi-parity" $\rho_{n, h}$ is any real or complex overlap between the eigenvectors before their biorthonormalization,

$$
\left\langle\left\langle n, h \mid n^{\prime}, h^{\prime}\right\rangle=\delta_{n, n^{\prime}} \delta_{h, h^{\prime}} \rho_{n, h} .\right.
$$

A priori, the spectra of the eigenvalues of the operators $H$ and/or $\Lambda$ which satisfy relations (49) may be either purely real or encompassing certain conjugate pairs of eigenvalues or containing certain "exceptional" points where the diagonalizability has been lost [11].

Whenever one contemplates the former option only, the reality of the eigenvalues opens a way towards a physical interpretation of the model where the eigenvalues of 
operators $H$ and $\Lambda$ may be observable and where the distribution of these eigenvalues can be experimentally measured. In the literature, a successful attempt of such a type has recently been completed for the spinless KleinGordon system [5]. In our present paper we made the first steps in a similar direction for spin one. For several alternative versions of Hamiltonian $H$ and associated helicity operator $\Lambda$ we constructed certain "sufficiently simple" operators $\mathcal{P}$ playing the role of an indefinite metric which characterizes the relativistic Proca field of the massive vector bosons with spin one.

\section{B. Outlook}

Our present study has been inspired by the well known fact that in the massless limit of the vector bosons a construction of the physical metric becomes trivial. In particular, the operator $\Theta$ of Eq. (2) degenerates to a projector in a way explained by Gupta and Bleuler [26] (cf. also Appendix A below). In this sense our present study could be perceived as a preparatory clarification of several $\mathcal{P} \mathcal{T}$-symmetry-related questions emerging during the canonical quantization of the massive model.
Even in the absence of interactions we may expect that the construction of the positively definite $\Theta$ will be much less easy than in the electromagnetic massless case. Its details seem to have represented an open problem up to now [18]. At the same time, the construction of $\Theta$ should be considered urgent at any spin. Its phenomenological relevance stems from the necessity of a search for a compromise between the complexity of the fieldtheoretical calculations and a comparative simplicity of the various pragmatic semi- or non-relativistic models of the bound states (say, in a pionic atom, etc). We believe that the challenge of the construction of $\Theta$ for spin one [25] might also re-open several new purely theoretical questions paralleling the ones encountered already in the zero-spin context of the Feshbach-Villars version of the first-quantized Klein-Gordon equation [5].

\section{ACKNOWLEDGEMENTS}

The participation of VJ and MZ was supported by IRP AV0Z10480505, as well as the "Doppler Institute" MSYS project Nr. LC06002 and the GAČR grant Nr. $202 / 07 / 1307$.

\section{APPENDIX A. PARALLELS BETWEEN MASSIVE SPIN-ONE BOSONS AND MASSLESS PHOTONS}

In a way resembling the description of the massless (i.e., electromagnetic) fields let us now change the notation and introduce an antisymmetric tensor $F^{\mu \nu}$ such that

$$
\begin{aligned}
& F^{i j}=-\varepsilon_{i j m} B_{m}, \\
& F^{0 j}=-E_{j}, \quad i, j=1,2,3 .
\end{aligned}
$$

In a fixed inertial frame equations (3) then acquire the form

$$
\begin{aligned}
& \mathbf{B}=\operatorname{rot} \mathbf{A}, \quad \frac{\partial \mathbf{A}}{\partial t}=-\mathbf{E}-\operatorname{grad} A_{0} \\
& A_{0}=-m^{-2} \operatorname{div} \mathbf{E}, \frac{\partial \mathbf{E}}{\partial t}=m^{2} \mathbf{A}+\operatorname{rot} \mathbf{B} .
\end{aligned}
$$

An elimination of $\mathbf{B}$ and $A_{0}$ gives

$$
\frac{\partial \mathbf{A}}{\partial t}=-\mathbf{E}+\frac{\nabla^{2} \mathbf{E}}{2 m^{2}}+\frac{\hat{q}}{2 m^{2}} \mathbf{E}, \quad \frac{\partial \mathbf{E}}{\partial t}=m^{2} \mathbf{A}-\frac{\nabla^{2} \mathbf{A}}{2}+\frac{\hat{q}}{2} \mathbf{A},
$$

where we abbreviated

$$
\hat{q}=2 \operatorname{grad} \operatorname{div}-\nabla^{2}
$$

Finally, another abbreviation

$$
\Phi^{T}=(\mathbf{u}, \mathbf{v}), \quad \mathbf{u}=\mathbf{E}+i m \mathbf{A}, \quad \mathbf{v}=\mathbf{E}-i m \mathbf{A}
$$

converts equations (56) into the six-component evolution rule

$$
\begin{aligned}
i \frac{\partial \Phi}{\partial t} & =\left(\begin{array}{cc}
m-\frac{\nabla^{2}}{2 m} & -\frac{\hat{q}}{2 m} \\
\frac{\hat{q}}{2 m} & -m+\frac{\nabla^{2}}{2 m}
\end{array}\right) \Phi \\
& =\left(\begin{array}{cc}
m-\frac{\nabla^{2}}{2 m} & -\frac{\nabla^{2}}{2 m}+\frac{(\mathbf{S} \nabla)^{2}}{m} \\
\frac{\nabla^{2}}{2 m}-\frac{(\mathbf{S} \nabla)^{2}}{m} & -m+\frac{\nabla^{2}}{2 m}
\end{array}\right) \Phi=H_{T S} \Phi .
\end{aligned}
$$


The matrix operator $H_{T S}$ is not Hermitian and fulfills the relation

$$
H_{T S}^{\dagger}=\sigma_{3} H_{T S} \sigma_{3}, \quad \sigma_{3}=\left(\begin{array}{cc}
I_{3} & 0 \\
0 & -I_{3}
\end{array}\right)
$$

shared by the spinless and spin-one cases $[3,27]$.

[1] W. Greiner, Relativistic Quantum Mechanics - Wave Equations (Springer, Berlin, 1997).

[2] A. Proca, Compt. Rend. 202, 1490 (1936); M. Yu. Kuchiev, V. V. Flambaum, Phys. Rev. D 73, 093009 (2006).

[3] H. Feshbach, F. Villars, Rev. Mod. Phys. 30, 24 (1958).

[4] F. Constantinescu, E. Magyari, Problems in Quantum Mechanics (Pergamon, Oxford, 1971).

[5] A. Mostafazadeh, J. Math. Phys. 44, 974 (2003); Ann. Phys. 309, 1 (2004).

[6] M. Znojil, Czechosl. J. Phys. 54, 151 (2004); 55, 1187 (2005); M. Znojil, in Symmetry Methods in Physics, edited by C. Burdik, O. Navratil, S. Posta (JINR, Dubna, 2004), CD ROM proc. series, hep-th/0408081; F. Kleefeld, Czechosl. J. Phys. 56, 999 (2006).

[7] C. M. Bender, S. Boettcher, Phys. Rev. Lett. 80, 4243 (1998).

[8] E. Caliceti, S. Graffi, M. Maioli, Commun. Math. Phys. 75, 51 (1980); N. Hatano, D. R. Nelson, Phys. Rev. Lett. 77, 570 (1996); C. M. Bender, K. A. Milton, Phys. Rev. D 55, R3255 (1997); F. M. Fernández et al, J. Phys. A 31, 10105 (1998); A. A. Andrianov, F. Cannata, JP. Dedonder, M. V. Ioffe, Int. J. Mod. Phys. A 14, 2675 (1999).

[9] M. Znojil, Phys. Lett. A 259, 220 (1999); E. Caliceti, F. Cannata, S. Graffi, J. Phys. 37, 2239 (2006); H. Bíla, Czech. J. Phys. 54, 1049 (2004); M. Znojil, J. Phys. 39, 10247 (2006).

[10] P. Dorey, C. Dunning, R. Tateo, J. Phys. 34, 5679 (2001); K. C. Shin, J. Math. Phys. 42, 2513 (2001).

[11] U. Günther, O. Kirillov, J. Phys. A 39, 10057 (2006).

[12] B. Bagchi, C. Quesne, M. Znojil, Mod. Phys. Lett. A 16, 2047 (2001); A. Mostafazadeh, J. Math. Phys. 43, 205
(2002); C. M. Bender, D. C. Brody, H. F. Jones, Phys. Rev. Lett. 89, 0270401 (2002); R. Kretschmer, L. Szymanowski, Phys. Lett. A 325112 (2004); H. Langer, C. Tretter, Czech. J. Phys. 54, 1113 (2004).

[13] M. Znojil, J. Phys. A 39, 441 (2006).

[14] F. G. Scholz, H. B. Geyer, Phys. Lett. B 634, 84 (2006).

[15] M. Znojil, Phys. Lett. A 353, 463 (2006).

[16] F. G. Scholz, H. B. Geyer, F. J. W. Hahne, Ann. Phys. 213, 74 (1992).

[17] M. Taketani, S. Sakata, Proc. Phys. Math. Soc. Jpn 22, 757 (1940); I. Tamm, Compt. Rend. Acad. Sci. (URSS) 29, 551 (1940).

[18] W. I. Fushchych, A. G. Nikitin, W. M. Susloparow, Nuovo Cimento 87, 415 (1985); W. I. Fushchych, A. G. Nikitin, Symmetries of Equations of Quantum Mechanics (Allerton Press, New York, 1994); A. G. Sergheyev, Ukr. J. Phys. 42, 1171 (1997).

[19] N. Kemmer, Proc. Roy. Soc. A 177, 9 (1939).

[20] D. Shay, R. H. Good, Phys. Rev. 179, 141 (1969); S. Weinberg, Phys. Rev. B 133, 1318 (1964).

[21] G. Labenté, Nuovo Cimento 80, 77 (1984).

[22] P. A. M. Dirac, Lectures on Quantum Mechanics (Yeshiva University Press, New York, 1964).

[23] M. Henneaux, C. Teitelboim, Quantization of Gauge Systems (UP, Princeton, 1994).

[24] D. M. Gitman, I. V. Tyutin, Quantization of Fields with Constraints (SpringerVerlag, New York, 1990).

[25] V. Jakubský, J. Smejkal, Czech. J. Phys. 56, 985 (2006).

[26] S. N. Gupta, Proc. Phys. Soc. Sect. A 63, 681 (1950); K. Bleuler, Helv. Phys. Acta 23, 567 (1950).

[27] K. M. Case, Phys. Rev. 95, 1323 (1954); F. S. G. von Zuben, J. Math. Phys. 41, 6093 (2000).

\title{
РЕЛЯТИВІСТСЬКІ ВЕКТОРНІ БОЗОНИ ТА РТ-СИМЕТРІЯ
}

\author{
Я. Смейкал ${ }^{1}$, В. Якубський ${ }^{2}$, М. Зноїл ${ }^{2}$ \\ ${ }^{1}$ Інститут техніки та експерименталъної фізики ЧВУТ, \\ ${ }^{2}$ Iнститут ядерної фізики АН ЧР
}

\begin{abstract}
Розглянуто кілька схем квантування релятивістських масивних бозонів зі спіном 1. Для цих схем було показано, що система описується неермітовим гамільтоніяном $H \neq H^{\dagger}$ та оператором $\Lambda$. Ми з'ясували, що в усіх розглянутих схемах оператори $H$ і $\Lambda$ є одночасно $\mathcal{P} \mathcal{T}$-симетричними, тобто псевдоермітовими стосовно певного, не надто складного, псевдометричного оператора $\mathcal{P}=\mathcal{P}^{\dagger}$.
\end{abstract}

